\title{
Contribution of QCD condensates to the OPE of Green functions of chiral currents
}

\author{
Tomáš Kadavý ${ }^{1, *}$, Karol Kampf ${ }^{1,2}$, and Jiří Novotný ${ }^{1}$ \\ ${ }^{1}$ Institute of Particle and Nuclear Physics, Charles University, Prague, Czech Republic \\ ${ }^{2}$ Department of Astronomy and Theoretical Physics, Lund University, Lund, Sweden
}

\begin{abstract}
A framework of operator product expansion (OPE) allows us to study high-energy behaviour of Green functions. A calculation of such Green functions within chiral perturbation theory $(\chi \mathrm{PT})$ or resonance chiral theory $(\mathrm{R} \chi \mathrm{T})$ and subsequent matching of the result to the OPE enables us to determine constraints for unknown parameters of the effective theories. We present such procedure for Green functions in the odd-intrinsic parity sector of QCD.
\end{abstract}

\section{Introduction}

The amplitudes of physical processes can be computed using the LSZ reduction formula from the Green functions, the vacuum expectation values of time ordered products of composite operators $O_{i}\left(x_{i}\right)$ (the group and Lorentz indices are suppressed):

$$
\Pi(p, q)=\int \mathrm{d}^{4} x \mathrm{~d}^{4} y e^{-i(p \cdot x+q \cdot y)}\left\langle 0\left|\mathrm{~T}\left[O_{1}(x) O_{2}(y) O_{3}(0)\right]\right| 0\right\rangle .
$$

Within our context, the operators $O_{i}\left(x_{i}\right)$ stand for any of the non-singlet vector $V_{\mu}^{a}(x)=$ $\bar{q}(x) \gamma_{\mu} T^{a} q(x)$ and axial-vector $A_{\mu}^{a}(x)=\bar{q}(x) \gamma_{\mu} \gamma_{5} T^{a} q(x)$ chiral currents or scalar $S^{a}(x)=$ $\bar{q}(x) T^{a} q(x)$ and pseudoscalar $P^{a}(x)=i \bar{q}(x) \gamma_{5} T^{a} q(x)$ densities. The flavor matrices $T^{a}$ are defined as halves of Gell-Mann matrices, $T^{a}=\lambda^{a} / 2$.

There are 15 nontrivial three-point Green functions. For clarity, we can separate them into two groups:

- Set I: $\langle A S P\rangle,\langle V S S\rangle,\langle V P P\rangle,\langle V V A\rangle,\langle A A A\rangle,\langle A A V\rangle,\langle V V V\rangle$.

- Set II: $\langle S S S\rangle,\langle S P P\rangle,\langle V V P\rangle,\langle A A P\rangle,\langle V A S\rangle,\langle V V S\rangle,\langle A A S\rangle,\langle V A P\rangle$.

Having to determine the behaviour of Green functions at high energies, OPE is a suitable framework. It says that at large external momenta (or, in QCD, at short distances), the Green function can be written down as a sum of Wilson coefficients proportional to vacuum averages of composite gauge-invariant local operators made of quark and gluon fields, i.e. the QCD condensates. In other words, for $(x, y) \rightarrow(0,0)$ we are allowed to write

$$
\begin{aligned}
\left\langle 0\left|O_{1}(x) O_{2}(y) O_{3}(0)\right| 0\right\rangle & =C_{\text {pert. }}+C_{\langle\bar{q} q\rangle}\langle 0|\bar{q} q| 0\rangle+C_{\left\langle G^{2}\right\rangle}\left\langle 0\left|G_{\mu \nu} G^{\mu v}\right| 0\right\rangle \\
& +C_{\langle\bar{q} G q\rangle}\left\langle 0\left|\bar{q} \sigma_{\mu \nu} G^{\mu v} q\right| 0\right\rangle+C_{\langle 4 q\rangle}\left\langle 0\left|\bar{q} X_{1} q \bar{q} X_{2} q\right| 0\right\rangle+\ldots
\end{aligned}
$$

\footnotetext{
*e-mail: kadavy@ipnp.troja.mff.cuni.cz
} 
The first term in Eq. (2) corresponds to the perturbative contribution and the subsequent ones stand for the quark, gluon, quark-gluon and four-quark condensates. The Wilson coefficients $C_{i}$ contain informations about short-distance physics, i.e. the dynamics above some scale $\mu$, and are calculable in perturbative QCD by means of Feynman diagrams.

In our work, we are mostly interested in Green functions that belong to the odd-intrinsic parity sector of QCD. Such Green functions are $\langle V V P\rangle,\langle A A P\rangle,\langle V A S\rangle$ and anomalous correlators $\langle V V A\rangle$ and $\langle A A A\rangle$. To calculate contribution of resonances to these Green functions, we use the NLO resonance Lagrangian, described in detail in Ref. [1].

\section{Example: $\langle V V A\rangle$ Green function}

The $\langle V V A\rangle$ Green function is an object with a paramount theoretical and phenomenological importance and as such serves as a good example of our study.

The Ward identities and Lorentz and parity invariance allows us to decompose the $\langle V V A\rangle$ correlator into four terms:

$$
\left[\Pi_{V V A}(p, q ; r)\right]_{\mu \nu \rho}^{a b c}=d^{a b c}\left(w_{L} \varepsilon_{\mu v(p)(q)} r_{\rho}+\sum_{i=1}^{3} w_{T}^{(i)}\left(p^{2}, q^{2}, r^{2}\right) \Pi_{\mu \nu \rho}^{(i)}(p, q, r)\right),
$$

where the first term is fixed entirely by the chiral anomaly and a specific form of the transversal tensors $\Pi_{\mu \nu \rho}^{(i)}$ can be found in Ref. [2]. Using the functions $w_{T}^{(i)}\left(p^{2}, q^{2}, r^{2}\right)$, one can define the formfactor $w_{T}\left(Q^{2}\right)$ :

$$
w_{T}\left(Q^{2}\right)=-16 \pi^{2}\left[w_{T}^{(1)}\left(-Q^{2}, 0,-Q^{2}\right)+w_{T}^{(3)}\left(-Q^{2}, 0,-Q^{2}\right)\right],
$$

for which we already know the result for OPE of $\left\langle A V^{\star} V\right\rangle$, obtained in Ref. [3], where one of the momenta is soft:

$$
w_{T}\left(Q^{2}\right)=\frac{N_{c}}{Q^{2}}+\frac{128 \pi^{3} \alpha_{s} \chi\langle\bar{q} q\rangle^{2}}{9 Q^{6}}+O\left(\frac{1}{Q^{8}}\right) .
$$

Using the mentioned $\mathrm{R} \chi \mathrm{T}$ Lagrangian, we can calculate contribution of resonances to the $\langle V V A\rangle$ at $O\left(p^{6}\right)$, in terms of the formfactors defined above. Our result [4] can be used to construct the formfactor $w_{T}\left(Q^{2}\right)$ and compared with Eq. (5), which leads to a prediction for some unknown coupling constants of the NLO resonance Lagrangian. For example, for one of the couplings, we have found $\kappa_{5}^{V A}=-0.086$, which can be also compared with the value obtained from the decay of the axial resonance $f_{1}(1285)$, which gives $\kappa_{5}^{V A}=-0.062 \pm 0.030$.

We are also able to predict the value for the deviation parameter, which describes by how much is the Brodsky-Lepage behaviour of the pion transition formfactor $\mathcal{F}_{\pi^{0} \gamma \gamma}^{\mathrm{R} \chi \mathrm{T}}$ violated. We found $\delta_{\mathrm{BL}}=-1.342$. Then, we have taken data sets for the measured pion transition formfactor, obtained by the BABAR [5], BELLE [6] and CLEO [7] collaborations, and compared them with the theoretical formula for the pion transition formfactor for various values of the deviation parameter. The comparison is depicted in Fig. 1, which shows clear disagreement between the predicted value $\delta_{\mathrm{BL}}=-1.342$ and experimental data.

Now we have concluded that for the $\langle V V A\rangle$ correlator, its OPE with one soft momentum does not lead to an agreement with experiments. However, there is no physical argument that this kind of OPE is the best choice to use. Therefore, our proposed solution is to calculate OPE with all three momenta large, instead of only two. We also include higher-order contributions of QCD condensates and use them once again to express couplings of the NLO resonance Lagrangian. 


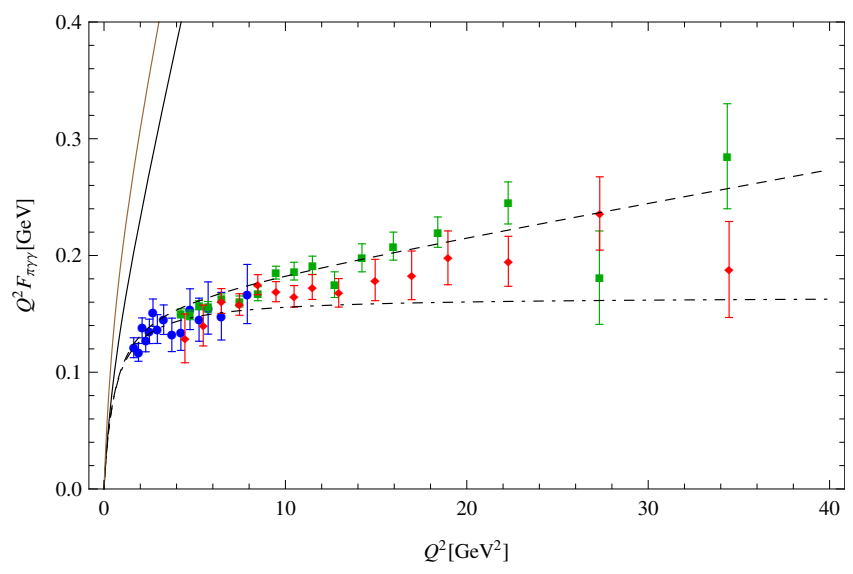

Figure 1. A plot of BABAR (green), BELLE (red) and CLEO (blue) data fitted with the formfactor $\mathcal{F}_{\pi^{0} \gamma \gamma}^{\mathrm{R} \chi \mathrm{T}}\left(0,-Q^{2} ; 0\right)$ using the modified Brodsky-Lepage behavior. The full black line represents $\mathcal{F}_{\pi^{0} \gamma \gamma}^{\mathrm{R} \chi \mathrm{T}}\left(0,-Q^{2} ; 0\right)$ with $\delta_{\mathrm{BL}}=-1.342$, the dashed line stands for $\delta_{\mathrm{BL}}=-0.055$ and the dot-dashed line for $\delta_{\mathrm{BL}}=0$. The full brown line is the LMD formfactor, which is very close to $\mathcal{F}_{\pi^{0} \gamma \gamma}^{\mathrm{R} T}\left(0,-Q^{2} ; 0\right)$ in the case of the $\delta_{\mathrm{BL}}$ value obtained from the OPE for $\left\langle A V^{\star} V\right\rangle$.

\section{OPE for all momenta large}

We have decided to calculate the contributions of the QCD condensates shown in Eq. (2) not only for the $\langle V V A\rangle$ Green function, but for all three-point correlators. After revisiting the results of the quark condensate [8, 9], we evaluated contribution of the gluon condensate, using the Fock-Schwinger gauge (FSG) [10].

Then we investigated contribution of the quark-gluon and four-quark condensates, which are given by propagation of nonlocal quark $\langle\bar{q}(x) q(y)\rangle$ and quark-gluon $\langle\bar{q}(x) \mathcal{A}(y) q(z)\rangle$ condensates, after expanding the quark and gluon fields in the FSG and extracting the local condensates.

Obviously, not every Green function has a nontrivial contribution from every QCD condensate. As it turns out, the perturbative contribution, gluon and four-quark condensates contribute to the correlators of the Set I. The quark and quark-gluon condensate, on the other hand, contribute to the Green functions of the Set II.

\section{Conclusion}

To conclude, our work is still ongoing, however, the final results of contributions of the QCD condensates to all three-point Green functions made of chiral currents will be published fairly soon in Ref. [4].

The work was supported by The Czech Science Foundation (project GAČR no. 18-17224S).

\section{References}

[1] K. Kampf et al., Phys. Rev. D 84, 014036 (2011)

[2] M. Knecht et al., JHEP 0403, 035 (2004)

[3] P. Colangelo et al., Phys. Rev. D 85, 035013 (2012)

[4] T. Kadavý et al. (in preparation)

[5] B. Aubert et al. [BaBar Collaboration], Phys. Rev. D 80, 052002 (2009)

[6] S. Uehara et al. [Belle Collaboration], Phys. Rev. D 86, 092007 (2012)

[7] J. Gronberg et al. [CLEO Collaboration], Phys. Rev. D 57, 33 (1998)

[8] B. Moussallam, Phys. Rev. D 51, 4939 (1995)

[9] M. Jamin et al., JHEP 0804, 040 (2008)

[10] V. A. Fock, Phys. Z. Sowjetunion 12, 404 (1937) 